\title{
A best-possible double inequality between Seiffert and harmonic means
}

\author{
Yu-Ming Chu ${ }^{1 *}$, Miao-Kun Wang ${ }^{1}$ and Zi-Kui Wang ${ }^{2}$
}

* Correspondence:

chuyuming2005@yahoo.com.cn

'Department of Mathematics,

Huzhou Teachers College, Huzhou, 313000, China

Full list of author information is available at the end of the article

\begin{abstract}
In this paper, we establish a new double inequality between the Seiffert and harmonic means.

The achieved results is inspired by the papers of Sándor (Arch. Math., 76, 34-40, 2001) and Hästö (Math. Inequal. Appl., 7, 47-53, 2004), and the methods from Wang et al. (J. Math. Inequal., 4, 581-586, 2010). The inequalities we obtained improve the existing corresponding results and, in some sense, are optimal.
\end{abstract}

2010 Mathematics Subject Classification: 26E60.

Keywords: harmonic mean, Seiffert mean, inequality

\section{Introduction}

For $a, b>0$ with $a \neq b$, the Seiffert mean $P(a, b)$ was introduced by Seiffert [1] as follows:

$$
P(a, b)=\frac{a-b}{4 \arctan \sqrt{a / b}-\pi} .
$$

Recently, the bivariate mean values have been the subject of intensive research. In particular, many remarkable inequalities for the Seiffert mean can be found in the literature [1-9].

Let $H(a, b)=2 a b /(a+b), G(a, b)=\sqrt{a b}, L(a, b)=(a-b) /(\log a-\log b), I(a, b)=1 / e$ $\left(b^{b} / a^{a}\right)^{1 /(b-a)}, A(a, b)=(a+b) / 2, C(a, b)=\left(a^{2}+b^{2}\right) /(a+b)$, and $M_{p}(a, b)=\left(\left(a^{p}+b^{p}\right) / 2\right)^{1 / p}$ $(p \neq 0)$ and $M_{0}(a, b)=\sqrt{a b}$ be the harmonic, geometric, logarithmic, identric, arithmetic, contraharmonic, and $p$-th power means of two different positive numbers $a$ and $b$, respectively. Then, it is well known that

$$
\begin{aligned}
\min \{a, b\}<H(a, b)= & M_{-1}(a, b)<G(a, b)=M_{0}(a, b)<L(a, b) \\
& <I(a, b)<A(a, b)=M_{1}(a, b)<C(a, b)<\max \{a, b\} .
\end{aligned}
$$

For all $a, b>0$ with $a \neq b$, Seiffert [1] established that $L(a, b)<P(a, b)<I(a, b)$; Jagers [4] proved that $M_{1 / 2}(a, b)<P(a, b)<M_{2 / 3}(a, b)$ and $M_{2 / 3}(a, b)$ is the bestpossible upper power mean bound for the Seiffert mean $P(a, b)$; Seiffert [7] established that $P(a, b)>A(a, b) G(a, b) / L(a, b)$ and $P(a, b)>2 A(a, b) / \pi$; Sándor [6] presented that $(A(a, b)+G(a, b)) / 2<P(a, b)<\sqrt{A(a, b)(A(a, b)+G(a, b)) / 2} \quad$ and $\sqrt[3]{A^{2}(a, b) G(a, b)}<P(a, b)<(G(a, b)+2 A(a, b)) / 3$; Hästö [3] proved that $P(a, b)>$

(c) 2011 Chu et al; licensee Springer. This is an Open Access article distributed under the terms of the Creative Commons Attribution License (http://creativecommons.org/licenses/by/2.0), which permits unrestricted use, distribution, and reproduction in any medium, provided the original work is properly cited. 
$M_{\log 2 / \log \pi}(a, b)$ and $M_{\log 2 / \log \pi}(a, b)$ is the best-possible lower power mean bound for the Seiffert mean $P(a, b)$.

Very recently, Wang and Chu [8] found the greatest value $\alpha$ and the least value $\beta$ such that the double inequality $A^{\alpha}(a, b) H^{1-\alpha}(a, b)<P(a, b)<A^{\beta}(a, b) H^{1-\beta}(a, b)$ holds for $a, b>0$ with $a \neq b$; For any $\alpha \in(0,1)$, Chu et al. [10] presented the best-possible bounds for $P^{\alpha}(a, b) G^{1-\alpha}(a, b)$ in terms of the power mean; In [2], the authors proved that the double inequality $\alpha A(a, b)+(1-\alpha) H(a, b)<P(a, b)<\beta A(a, b)+(1-\beta) H(a$, $b$ ) holds for all $a, b>0$ with $a \neq b$ if and only if $\alpha \leq 2 / \pi$ and $\beta \geq 5 / 6$; Liu and Meng [5] proved that the inequalities

$$
\alpha_{1} C(a, b)+\left(1-\alpha_{1}\right) G(a, b)<P(a, b)<\beta_{1} C(a, b)+\left(1-\beta_{1}\right) G(a, b)
$$

and

$$
\alpha_{2} C(a, b)+\left(1-\alpha_{2}\right) H(a, b)<P(a, b)<\beta_{2} C(a, b)+\left(1-\beta_{2}\right) H(a, b)
$$

hold for all $a, b>0$ with $a \neq b$ if and only if $\alpha_{1} \leq 2 / 9, \beta_{1} \geq 1 / \pi, \alpha_{2} \leq 1 / \pi$ and $\beta_{2} \geq 5 /$ 12.

For fixed $a, b>0$ with $a \neq b$ and $x \in[0,1 / 2]$, let

$$
h(x)=H(x a+(1-x) b, x b+(1-x) a) .
$$

Then, it is not difficult to verify that $h(x)$ is continuous and strictly increasing in [0, $1 / 2]$. Note that $h(0)=H(a, b)<P(a, b)$ and $h(1 / 2)=A(a, b)>P(a, b)$. Therefore, it is natural to ask what are the greatest value $\alpha$ and least value $\beta$ in $(0,1 / 2)$ such that the double inequality $H(\alpha a+(1-\alpha) b, \alpha b+(1-\alpha) a)<P(a, b)<H(\beta a+(1-\beta) b, \beta b+(1$ - $\beta$ )a) holds for all $a, b>0$ with $a \neq b$. The main purpose of this paper is to answer these questions. Our main result is the following Theorem 1.1.

Theorem 1.1. If $\alpha, \beta \in(0,1 / 2)$, then the double inequality

$$
H(\alpha a+(1-\alpha) b, \alpha b+(1-\alpha) a)<P(a, b)<H(\beta a+(1-\beta) b, \beta b+(1-\beta) a)
$$

holds for all $a, b>0$ with $a \neq b$ if and only if $\alpha \leq(1-\sqrt{1-2 / \pi}) / 2$ and $\beta \geq(6-\sqrt{6}) / 12$.

\section{Proof of Theorem 1.1}

Proof of Theorem 1.1. Let $\lambda=(1-\sqrt{1-2 / \pi}) / 2$ and $\mu=(6-\sqrt{6}) / 12$. We first prove that inequalities

$$
P(a, b)>H(\lambda a+(1-\lambda) b, \lambda b+(1-\lambda) a)
$$

and

$$
P(a, b)<H(\mu a+(1-\mu) b, \mu b+(1-\mu) a)
$$

hold for all $a, b>0$ with $a \neq b$.

Without loss of generality, we assume that $a>b$. Let $t=\sqrt{a / b}>1$ and $p \in(0,1 / 2)$; then, from (1.1), one has 


$$
\begin{aligned}
& H(p a+(1-p) b, p b+(1-p) a)-P(a, b) \\
& \quad=\frac{2\left[p t^{2}+(1-p)\right]\left[(1-p) t^{2}+p\right]}{t^{2}+1}-\frac{t^{2}-1}{4 \arctan t-\pi} \\
& \quad=\frac{2\left[p t^{2}+(1-p)\right]\left[(1-p) t^{2}+p\right]}{\left(t^{2}+1\right)(4 \arctan t-\pi)} \\
& \quad \times\left\{4 \arctan t-\frac{t^{4}-1}{2\left[p t^{2}+(1-p)\right]\left[(1-p) t^{2}+p\right]}-\pi\right\} .
\end{aligned}
$$

Let

$$
f(t)=4 \arctan t-\frac{t^{4}-1}{2\left[p t^{2}+(1-p)\right]\left[(1-p) t^{2}+p\right]}-\pi,
$$

then, simple computations lead to

$$
\begin{aligned}
& f(1)=0, \\
& \lim _{t \rightarrow+\infty} f(t)=\pi-\frac{1}{2 p(1-p)}
\end{aligned}
$$

and

$$
f^{\prime}(t)=\frac{f_{1}(t)}{\left(t^{2}+1\right)\left[p(1-p) t^{4}+\left(2 p^{2}-2 p+1\right) t^{2}+p(1-p)\right]^{2}}
$$

where

$$
\begin{aligned}
f_{1}(t)=4 p^{2} & (1-p)^{2} t^{8}-\left(2 p^{2}-2 p+1\right) t^{7}+8 p(1-p)\left(2 p^{2}-2 p+1\right) t^{6} \\
& +\left(2 p^{2}-2 p-1\right) t^{5}+4\left(6 p^{4}-12 p^{3}+10 p^{2}-4 p+1\right) t^{4} \\
& +\left(2 p^{2}-2 p-1\right) t^{3}+8 p(1-p)\left(2 p^{2}-2 p+1\right) t^{2} \\
& -\left(2 p^{2}-2 p+1\right) t+4 p^{2}(1-p)^{2} .
\end{aligned}
$$

Note that

$$
\begin{aligned}
& f_{1}(1)=0 \text {, } \\
& \lim _{t \rightarrow+\infty} f_{1}(t)=+\infty \\
& {f^{\prime}}^{\prime}(t)=32 p^{2}(1-p)^{2} t^{7}-7\left(2 p^{2}-2 p+1\right) t^{6}+48 p(1-p)\left(2 p^{2}-2 p+1\right) t^{5} \\
& +5\left(2 p^{2}-2 p-1\right) t^{4}+16\left(6 p^{4}-12 p^{3}+10 p^{2}-4 p+1\right) t^{3} \\
& +3\left(2 p^{2}-2 p-1\right) t^{2}+16 p(1-p)\left(2 p^{2}-2 p+1\right) t \\
& -\left(2 p^{2}-2 p+1\right) \\
& f_{1}^{\prime}(1)=0, \\
& \lim _{t \rightarrow+\infty} f_{1}^{\prime}(t)=+\infty .
\end{aligned}
$$

Let $f_{2}(t)=f_{1}^{\prime \prime}(t) / 2, f_{3}(t)=f_{2}^{\prime}(t) / 3, f_{4}(t)=f_{3}^{\prime}(t) / 4, f_{5}(t)=f_{4}^{\prime}(t) / 5, f_{6}(t)=f_{5}^{\prime}(t) / 6$ and $f_{7}(t)=f_{6}^{\prime}(t) / 7$. Then, simple computations lead to 


$$
\begin{aligned}
& f_{2}(t)=112 p^{2}(1-p)^{2} t^{6}-21\left(2 p^{2}-2 p+1\right) t^{5}+120 p(1-p)\left(2 p^{2}-2 p+1\right) t^{4} \\
& +10\left(2 p^{2}-2 p-1\right) t^{3}+24\left(6 p^{4}-12 p^{3}+10 p^{2}-4 p+1\right) t^{2} \\
& +3\left(2 p^{2}-2 p-1\right) t+8 p(1-p)\left(2 p^{2}-2 p+1\right) \text {, } \\
& f_{2}(1)=-2\left(24 p^{2}-24 p+5\right) \\
& \lim _{t \rightarrow+\infty} f_{2}(t)=+\infty \\
& f_{3}(t)=224 p^{2}(1-p)^{2} t^{5}-35\left(2 p^{2}-2 p+1\right) t^{4}+160 p(1-p)\left(2 p^{2}-2 p+1\right) t^{3} \\
& +10\left(2 p^{2}-2 p-1\right) t^{2}+16\left(6 p^{4}-12 p^{3}+10 p^{2}-4 p+1\right) t \\
& +\left(2 p^{2}-2 p-1\right) \text {, } \\
& f_{3}(1)=-6\left(24 p^{2}-24 p+5\right) \\
& \lim _{t \rightarrow+\infty} f_{3}(t)=+\infty \\
& f_{4}(t)=280 p^{2}(1-p)^{2} t^{4}-35\left(2 p^{2}-2 p+1\right) t^{3}+120 p(1-p)\left(2 p^{2}-2 p+1\right) t^{2} \\
& +5\left(2 p^{2}-2 p-1\right) t+4\left(6 p^{4}-12 p^{3}+10 p^{2}-4 p+1\right), \\
& f_{4}(1)=4\left(16 p^{4}-32 p^{3}-25 p^{2}+41 p-9\right), \\
& \lim _{t \rightarrow+\infty} f_{4}(t)=+\infty \\
& f_{5}(t)=224 p^{2}(1-p)^{2} t^{3}-21\left(2 p^{2}-2 p+1\right) t^{2}+48 p(1-p)\left(2 p^{2}-2 p+1\right) t \\
& +\left(2 p^{2}-2 p-1\right) \text {, } \\
& f_{5}(1)=2\left(64 p^{4}-128 p^{3}+20 p^{2}+44 p-11\right), \\
& \lim _{t \rightarrow+\infty} f_{5}(t)=+\infty \\
& f_{6}(t)=112 p^{2}(1-p)^{2} t^{2}-7\left(2 p^{2}-2 p+1\right) t \\
& +8 p(1-p)\left(2 p^{2}-2 p+1\right) \\
& f_{6}(1)=96 p^{4}-192 p^{3}+74 p^{2}+22 p-7 \\
& \lim _{t \rightarrow+\infty} f_{6}(t)=+\infty \\
& f_{7}(t)=32 p^{2}(1-p)^{2} t-\left(2 p^{2}-2 p+1\right)
\end{aligned}
$$

and

$$
f_{7}(1)=32 p^{4}-64 p^{3}+30 p^{2}+2 p-1 .
$$

We divide the proof into two cases. 
Case 1. $p=\lambda=(1-\sqrt{1-2 / \pi}) / 2$. Then equations (2.6), (2.13), (2.15), (2.17), (2.19), (2.22) and (2.25) become

$$
\begin{aligned}
& \lim _{t \rightarrow+\infty} f(t)=0, \\
& f_{2}(1)=-\frac{2(5 \pi-12)}{\pi}<0, \\
& f_{3}(1)=-\frac{6(5 \pi-12)}{\pi}<0, \\
& f_{4}(1)=-\frac{2\left(18 \pi^{2}-41 \pi-8\right)}{\pi^{2}}<0, \\
& f_{5}(1)=-\frac{2\left(11 \pi^{2}-22 \pi-16\right)}{\pi^{2}}<0, \\
& f_{6}(1)=-\frac{7 \pi^{2}-11 \pi-24}{\pi^{2}}<0
\end{aligned}
$$

and

$$
f_{7}(1)=\frac{\pi+8-\pi^{2}}{\pi^{2}}>0 .
$$

From (2.24), we clearly see that $f_{7}(t)$ is strictly increasing in $[1,+\infty)$, and then $(2.32)$ leads to the conclusion that $f_{7}(t)>0$ for $t \in[1,+\infty)$. Thus, $f_{6}(t)$ is strictly increasing in $[1,+\infty)$.

It follows from (2.23) and (2.31) together with the monotonicity of $f_{6}(t)$ that there exists $t_{1}>1$ such that $f_{6}(t)<0$ for $t \in\left(1, t_{1}\right)$ and $f_{6}(t)>0$ for $t \in\left(t_{1},+\infty\right)$. Thus, $f_{5}(t)$ is strictly decreasing in $\left[1, t_{1}\right]$ and strictly increasing in $\left[t_{1},+\infty\right)$.

From (2.20) and (2.30), together with the piecewise monotonicity of $f_{5}(t)$, we clearly see that there exists $t_{2}>t_{1}>1$ such that $f_{4}(t)$ is strictly decreasing in $\left[1, t_{2}\right]$ and strictly increasing in $\left[t_{2},+\infty\right)$. Then, equation (2.18) and inequality (2.29) lead to the conclusion that there exists $t_{3}>t_{2}>1$ such that $f_{3}(t)$ is strictly decreasing in $\left[1, t_{3}\right]$ and strictly increasing in $\left[t_{3},+\infty\right)$.

It follows from (2.16) and (2.28) together with the piecewise monotonicity of $f_{3}(t)$ we conclude that there exists $t_{4}>t_{3}>1$ such that $f_{2}(t)$ is strictly decreasing in $\left[1, t_{4}\right]$ and strictly increasing in $\left[t_{4},+\infty\right)$. Then, equation (2.14) and inequality (2.27) lead to the conclusion that there exists $t_{5}>t_{4}>1$ such that $f_{1}^{\prime}(t)$ is strictly decreasing in $\left[1, t_{5}\right]$ and strictly increasing in $\left[t_{5},+\infty\right)$.

From equations (2.11) and (2.12), together with the piecewise monotonicity of $f_{1}^{\prime}(t)$, we know that there exists $t_{6}>t_{5}>1$ such that $f_{1}(t)$ is strictly decreasing in $\left[1, t_{6}\right]$ and strictly increasing in $\left[t_{6},+\infty\right)$. Then, equations (2.7)-(2.10) lead to the conclusion that there exists $t_{7}>t_{6}>1$ such that $f(t)$ is strictly decreasing in $\left[1, t_{7}\right]$ and strictly increasing in $\left[t_{7},+\infty\right)$.

Therefore, inequality (2.1) follows from equations (2.3)-(2.5) and (2.26) together with the piecewise monotonicity of $f(t)$. 
Case 2. $p=\mu=(6-\sqrt{6}) / 12$. Then, equations (2.13), (2.15), (2.17), (2.19) and (2.21) become

$$
\begin{aligned}
& f_{2}(1)=0, \\
& f_{3}(1)=0, \\
& f_{4}(1)=\frac{17}{18}>0, \\
& f_{5}(1)=\frac{17}{9}>0
\end{aligned}
$$

and

$$
f_{6}(t)=\frac{1}{36}\left(175 t^{2}-147 t+35\right)>0
$$

for $t>1$.

From inequality (2.37), we know that $f_{5}(t)$ is strictly increasing in $[1,+\infty)$, and then inequality (2.36) leads to the conclusion that $f_{5}(t)>0$ for $t \in[1,+\infty)$. Thus, $f_{4}(t)$ is strictly increasing in $[1,+\infty)$.

It follows from inequality (2.35) and the monotonicity of $f_{4}(t)$ that $f_{3}(t)$ is strictly increasing in $[1,+\infty)$.

Therefore, inequality (2.2) follows easily from equations (2.3)-(2.5), (2.7), (2.9), (2.11), (2.33), and (2.34) together with the monotonicity of $f_{3}(t)$.

Next, we prove that $\lambda=(1-\sqrt{1-2 / \pi}) / 2$ is the best-possible parameter such that inequality (2.1) holds for all $a, b>0$ with $a \neq b$. In fact, if $(1-\sqrt{1-2 / \pi}) / 2=\lambda<p<1 / 2$, then equation (2.6) leads to

$$
\lim _{t \rightarrow+\infty} f(t)=\pi-\frac{1}{2 p(1-p)}>0 .
$$

Inequality (2.38) implies that there exists $T=T(p)>1$ such that

$$
f(t)>0
$$

for $t \in(T,+\infty)$.

From equations (2.3) and (2.4), together with inequality (2.39), we clearly see that $P$ $(a, b)<H(p a+(1-p) b, p b+(1-p) a)$ for $a / b \in\left(T^{2},+\infty\right)$.

Finally, we prove that $\mu=(6-\sqrt{6}) / 12$ is the best-possible parameter such that inequality (2.2) holds for all $a, b>0$ with $a \neq b$. In fact, if $0<p<\mu=(6-\sqrt{6}) / 12$, then equation (2.13) leads to

$$
f_{2}(1)=-2\left(24 p^{2}-24 p+5\right)<0 .
$$

Inequality (2.40) implies that there exists $\delta=\delta(p)>0$ such that

$$
f_{2}(t)<0
$$

for $t \in(1,1+\delta)$.

Therefore, $P(a, b)>H(p a+(1-p) b, p b+(1-p) a)$ for $a / b \in\left(1,(1+\delta)^{2}\right)$ follows from equations (2.3)-(2.5), (2.7), (2.9), and (2.11) together with inequality (2.41). 


\section{Acknowledgements}

This study is partly supported by the Natural Science Foundation of China (Grant no. 11071069), the Natural Science Foundation of Hunan Province (Grant no. 09J J6003), and the Innovation Team Foundation of the Department of Education of Zhejiang Province(Grant no. T200924).

\section{Author details}

'Department of Mathematics, Huzhou Teachers College, Huzhou, 313000, China ${ }^{2}$ Department of Mathematics, Hangzhou Normal University, Hangzhou, 310012, China

\section{Authors' contributions}

Y-MC provided the main idea in this paper. M-KW carried out the proof of inequality (2.1) in this paper. Z-KW carried out the proof of inequality (2.2) in this paper. All authors read and approved the final manuscript.

\section{Competing interests}

The authors declare that they have no competing interests.

Received: 12 June 2011 Accepted: 26 October 2011 Published: 26 October 2011

\section{References}

1. Seiffert, H-J: Problem 887. Nieuw Arch Wisk (4). 11(2), 176-176 (1993)

2. Chu, Y-M, Qiu, Y-F, Wang, M-K, Wang, G-D: The optimal convex combination bounds of arithmetic and harmonic means for the Seiffert's mean. J Inequal Appl2010, 7. Article ID 436457

3. Hästö, PA: Optimal inequalities between Seiffert's mean and power means. Math Inequal Appl. 7(1), 47-53 (2004)

4. Jagers, AA: Solution of Problem 887. Nieuw Arch Wisk (4). 12, 230-231 (1994)

5. Liu, H, Meng, X-J: The optimal convex combination bounds for Seiffert's mean. J Inequal Appl2011, 9. Article ID 686834

6. Sándor, J: On certain inequalities for means III. Arch Math. 76(1), 34-40 (2001). doi:10.1007/s000130050539

7. Seiffert, H-J: Ungleichungen für einen bestimmten Mittelwert. Nieuw Arch Wisk (4). 13(2), 195-198 (1995)

8. Wang, S-S, Chu, Y-M: The best bounds of the combination of arithmetic and harmonic means for the Seiffert's mean. Int J Math Anal. 4(21-24), 1079-1084 (2010)

9. Wang, M-K, Qiu, Y-F, Chu, Y-M: Sharp bounds for Seiffert means in terms of Lehmer means. J Math Inequal. 4(4), $581-586$ (2010)

10. Chu, Y-M, Qiu, Y-F, Wang, M-K: Sharp power mean bounds for the combination of Seiffert and geometric means. Abstr Appl Anal2010, 12. Article ID 108920

Cite this article as: Chu et al:: A best-possible double inequality between Seiffert and harmonic means. Journal of Inequalities and Applications 2011 2011:94.

\section{Submit your manuscript to a SpringerOpen ${ }^{\odot}$ journal and benefit from:}

- Convenient online submission

- Rigorous peer review

- Immediate publication on acceptance

- Open access: articles freely available online

- High visibility within the field

- Retaining the copyright to your article

Submit your next manuscript at $\boldsymbol{\nabla}$ springeropen.com 